Old Dominion University

ODU Digital Commons

2012

\title{
Anchoring of Drifting Spiral and Scroll Waves to Impermeable Inclusions in Excitable Media
}

Christian W. Zemlin

Old Dominion University, zemlinc@wustl.edu

Arkady M. Pertsov

Follow this and additional works at: https://digitalcommons.odu.edu/bioelectrics_pubs

Part of the Biomedical Engineering and Bioengineering Commons

\section{Original Publication Citation}

Zemlin, C. W., \& Pertsov, A. M. (2012). Anchoring of drifting spiral and scroll waves to impermeable inclusions in excitable media. Phys Rev Lett, 109(3), 038303. 


\title{
Anchoring of Drifting Spiral and Scroll Waves to Impermeable Inclusions in Excitable Media
}

\author{
Christian W. Zemlin \\ Department of Electrical and Computer Engineering, Old Dominion University, Norfolk, Virginia 23528, USA \\ and Frank Reidy Research Center for Bioelectrics, Norfolk, Virginia 23508, USA
}

Arkady M. Pertsov

Department of Pharmacology, SUNY Upstate Medical University, Syracuse, New York 13210, USA (Received 18 January 2011; revised manuscript received 10 April 2012; published 20 July 2012)

\begin{abstract}
Anchoring of spiral and scroll waves in excitable media has attracted considerable interest in the context of cardiac arrhythmias. Here, by bombarding inclusions with drifting spiral and scroll waves, we explore the forces exerted by inclusions onto an approaching spiral and derive the equations of motion governing spiral dynamics in the vicinity of inclusion. We demonstrate that these forces nonmonotonically depend on distance and can lead to complex behavior: (a) anchoring to small but circumnavigating larger inclusions; (b) chirality-dependent anchoring.
\end{abstract}

DOI: 10.1103/PhysRevLett.109.038303

PACS numbers: 82.40.Ck, 05.65.+b, 89.75.Kd

Excitable media encompass a broad class of highly nonlinear, distributed nonequilibrium systems of physical, chemical, and biological systems [1], including nerve and cardiac tissues [2]. A distinct feature of excitable media is their ability to support self-sustained waves and highfrequency sources of repetitive excitation in the form of rotating spiral waves (in 2D) [3,4] and scroll waves (in 3D) [5].

Rotating waves are organized around vortexlike cores that are called filaments in the case of scroll waves [6,7]. Spiral waves behave in many ways like particles: they can drift $[2,8,9]$, interact, and form dynamic bound states [10-12].

Drifting spiral and scroll waves can also anchor (pin) to localized heterogeneities, giving rise to sustained periodic or quasiperiodic activity [2,13-16]. Anchoring is believed to play a crucial role in maintaining abnormal highfrequency heart rhythms, including ventricular tachycardia and fibrillation, the main causes of sudden cardiac death [2]. The dynamics of anchored spirals, particularly methods of unpinning them, have been studied extensively with regard to cardiological applications [17-19].

In this study, we focus on impermeable inclusions, which in a cardiac context represent tissue that is electrically uncoupled from the excitable myocardium, e.g., blood vessels or scars. Unlike parametric heterogeneities, impermeable inclusions represent a different class of heterogeneities that reflect the topological connectivity of the excitable medium. Despite the importance of impermeable inclusions, their anchoring ability is much less understood. This is in part is due to their fundamentally nonperturbative nature, which prevents the application of analytical tools developed for parametric heterogeneities [16,20,21].

We derive the equation of motion of a spiral wave core in the vicinity of an impermeable inclusion by adopting a particle-physics approach. We bombard the inclusions with spiral wave cores of different speeds and impact parameters. By analyzing perturbations of drift trajectories we derive forces controlling the dynamics of the core in the vicinity of inclusion. Such "scattering experiments" are carried out numerically using a generic reaction-diffusion model of an excitable medium with Barkley kinetics [22],

$$
\begin{gathered}
\partial_{t} u=\epsilon^{-1} u(1-u)[u-(v+b) / a]+D \nabla^{2} u+\mathbf{E} \cdot \nabla u \\
\partial_{t} v=u-v+\alpha \mathbf{E} \cdot \nabla v,
\end{gathered}
$$

where $u$ is the activator variable, $v$ is the inhibitor variable, $D$ is the diffusivity, and $\mathbf{E}$ is an external field. The advection term $\mathbf{E} \cdot \nabla u$ was introduced to make spirals drift towards the inclusion. The parameter $\alpha$ is used to switch advection of $v$ on $(\alpha=1)$ or off $(\alpha=0)$.

Without loss of generality, we assumed that $\mathbf{E}$ is oriented along the $y$ axis. No-flux boundary conditions were set both at the border of the inclusion and at external medium boundaries. The values of the kinetic parameters $a, b$, and $\epsilon$ are given in the caption of Fig. 1; they were chosen such that in an unperturbed system $(\mathbf{E}=0)$, the spiral rotation was stationary and scroll wave filaments had positive tension [7]. We induced spiral and scroll waves as previously described [12]. To determine drift velocity, we tracked the movement of the spiral core, defined as the region with $u<0.99 u_{\max }$ over one spiral rotation period $(T)$. We use the spiral wavelength $\lambda$ as a natural unit of length.

Our numerical experiments show that the dynamics of a filament or spiral core approaching an inclusion can be complex and sometimes counterintuitive. For example, we found that in the same excitable medium, drifting filament or spiral cores anchored to small inclusions but always circumnavigated larger inclusions.

Figures 1(a) and 1(b) show an example of a 3D numerical experiment, in which a drifting filament has been set on 




FIG. 1. Dynamics of a drifting scroll wave filament in the vicinity of impermeable inclusions. Superposition of sequential filament positions, every 12 rotations. Numbers above the filament show the time after the initiation in rotation periods. (a) Anchoring to a small inclusion $(\rho=0.05 \lambda)$. (b) Filament avoiding a larger inclusion (radius $\rho=0.1 \lambda$ ). The values of impact parameter and field-induced drift velocity $\left|\mathbf{v}_{\mathbf{E}}\right|$ in (a) and (b) are the same $\left(\Delta=0,\left|\mathbf{v}_{\mathbf{E}}\right|=0.007 \lambda / T\right)$. Medium parameters are $\epsilon=0.02, a=0.9, b=0.05,120 \times 120 \times 120$ nodes, $\Delta x=0.25, \alpha=0$, and $D=0.5$.

a central collision course (impact parameter $\Delta=0$ ) with inclusions of two different sizes: first with a smaller radius $(\rho=0.05 \lambda)$ and then with a larger radius $(\rho=0.1 \lambda)$. In both cases, the filament initially drifts at a constant speed $\mathbf{v}_{\mathbf{E}}$ and preserves its initial rectilinear shape. As the filament gets closer to the inclusion, it undergoes significant deformation and changes its dynamics, which suggests the presence of strong forces exerted by the inclusion. In both cases, the filament bulges away from the inclusion, stalls, and starts turning clockwise. However, the subsequent evolution is very different. If $\rho$ is small [Fig. 1(a)], the turning suddenly accelerates (rotations 48-60) and the central part of the filament abruptly anchors. After the anchoring, the free ends of the filament continue to drift until they reach steady state (rotation 120). In contrast, if $\rho$ is larger, the filament does not anchor [Fig. 1(b)]. Instead, it circumnavigates the inclusion and resumes its drift in the original direction, gradually recovering its original rectilinear shape as it moves away from the inclusion.

A similar effect in a 2D system is illustrated in Figs. 2(a) and 2(b). As in the 3D case, the spiral core anchors to a smaller inclusion [Fig. 2(a)] but circumnavigates a larger one, resuming its drift in the original direction [see Fig. 2(b)]. This behavior is robust; we could reproduce it in both $2 \mathrm{D}$ and $3 \mathrm{D}$ for other values of the kinetic parameters $a$ and $b$ within the "contracting scroll" region [23] in the parameter space of the Barkley model.

As we show below in the 2D case, the motion of the spiral wave core in the vicinity of impermeable circular inclusion is governed by the following simple equation:

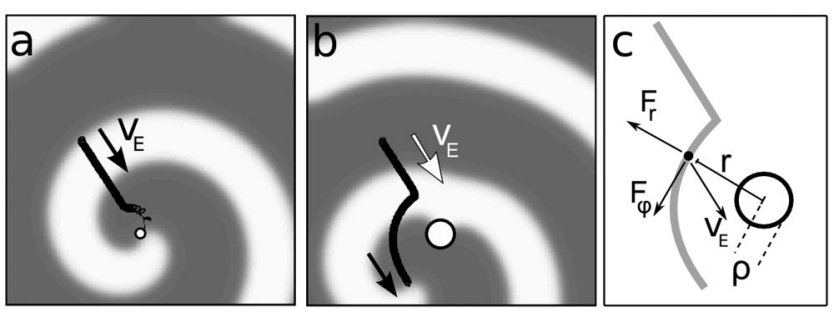

FIG. 2. Perturbation of a spiral drift trajectory by an impermeable inclusions of different sizes in the 2D case. Gray levels show the distribution of $u$ (dark gray: $u \approx 0$, white: $u \approx 1$ ). White circles mark the inclusions and the black lines show the spiral tip trajectory. (a) Small inclusion $(\rho=0.025 \lambda)$. (b) Larger inclusion $(\rho=0.1 \lambda)$. (c) Schematic showing the forces governing the dynamics of the spiral core in the vicinity of inclusion. Medium parameters as in Fig. $1,240 \times 240$ nodes, $\Delta x=0.125$, $\alpha=0$; see text for meaning of symbols.

$$
\begin{gathered}
\frac{d \mathbf{x}}{d t}=\mathbf{v}_{\mathbf{E}}+\mathbf{F}\left(r^{\prime}, \rho\right) \\
r^{\prime}=\left|\mathbf{x}-\mathbf{x}_{0}\right|-\rho,
\end{gathered}
$$

where $\mathbf{x}(t)$ is the center of the spiral wave core at time $t, \mathbf{v}_{\mathbf{E}}$ is the gradient-induced drift velocity, $r^{\prime}$ is the distance between the spiral core and the boundary of the inclusion, and $\mathbf{F}$ is the force that the inclusion exerts on the spiral core. $\mathbf{F}$ has a dimension of velocity, analogous to a Stokes force in a viscous medium; see also Refs. [16,20].

As in the case of localized parametric heterogeneities [16], $\mathbf{F}$ has a non-zero azimuthal component $\mathbf{F}_{\phi}$, which induces orbital drift of the spiral around the inclusion [see Fig. 2(c)]:

$$
\mathbf{F}=\mathbf{F}_{\mathbf{r}}+\mathbf{F}_{\phi}=F_{r} \mathbf{e}_{\mathbf{r}}+F_{\phi} \mathbf{e}_{\phi} .
$$

To validate Eqs. (3)-(5), we conducted a series of numerical experiments in which we systematically varied $\Delta,|\mathbf{E}|$, and $\rho$. Figure 3 shows two families of drift trajectories for $\left|\mathbf{v}_{\mathbf{E}}\right|=0.005$ and $\left|\mathbf{v}_{\mathbf{E}}\right|=0.01$ and $\Delta$ ranging from $-0.3 \lambda$ to $0.3 \lambda(\rho=0.16)$. For $\left|\mathbf{v}_{\mathbf{E}}\right|=0.005$, spirals initiated far from the inclusion never anchor [see Fig. 3(a)]: they circumnavigate the inclusion either from the left (trajectories -5 to -2 ) or from the right (trajectories -1 to 5 ). Increasing $\left|\mathbf{v}_{\mathbf{E}}\right|$ makes anchoring possible [see panel (b)]. Trajectories -1 and -2 , which were deflected from the anchor in panel (a), now anchor.

It is interesting that the spiral wave fails to anchor when on the central collision course with an inclusion $(\Delta=0)$ but anchors when its trajectory is significantly off center (trajectories -1 and -2 ). Significant asymmetry is also visible in Fig. 3(a) (the separatrix shown with a dashed line is shifted well to the left). This asymmetry is determined by the chirality of the spiral. In this particular experiment, the spiral rotated clockwise $(\mathrm{CW})$. For a counterclockwise $(\mathrm{CCW})$ rotating spiral, all trajectories are reflected at the 


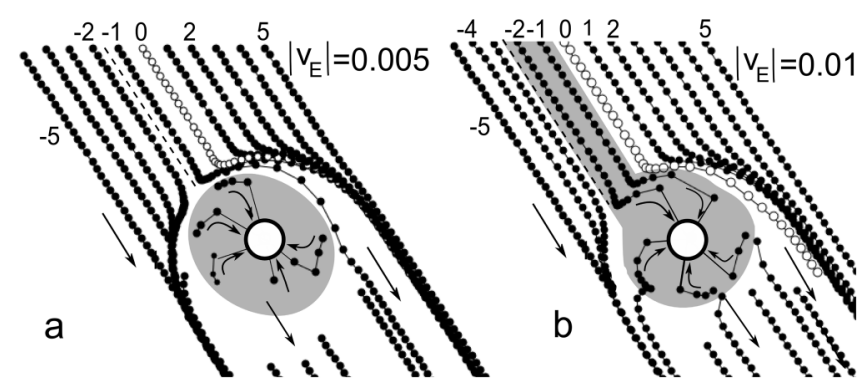

FIG. 3. Families of drift trajectories in the vicinity of an inclusion at subcritical (a) and supercritical (b) field strengths. Parameters of the medium and the grid size are as in Fig. 2. Arrows indicate drift direction. Dots show the spiral center every 3 rotations. Gray areas show the basin of attraction of the inclusion (white circle in the center). Dashed lines mark separatrices. Trajectory labels $(-5, \ldots, 5)$ indicate the impact parameter $\Delta$ for each trajectory in units of $0.06 \lambda$. (a) Subcritical field $\left(\left|\mathbf{v}_{\mathbf{E}}\right|=0.005 \lambda / T\right)$. Spiral waves initiated far from the inclusions do not anchor at any value of $\Delta$, and spirals initiated at $\left|\mathbf{x}-\mathbf{x}_{\mathbf{0}}\right|<0.2 \lambda$, in the direct vicinity of the inclusion, always anchor. (b) Supercritical drift velocity $\left(\left|\mathbf{v}_{\mathbf{E}}\right|=0.01 \lambda / T\right)$. Trajectory $0(\Delta=0)$ is marked by empty circles.

line parallel to $\mathbf{v}_{\mathbf{E}}$ and cutting through the center of the inclusion.

To demonstrate that Eq. (3) is correct, we did the following for a large variety of trajectories. We calculated $d \mathbf{x} / d t$ at different points for each trajectory. Then, using Eqs. (3)-(5) we derived $F_{r}$ and $F_{\phi}$ for each point and corrected them for time lag, which gave us $F_{r}\left(r^{\prime}\right)$ and $F_{\phi}\left(r^{\prime}\right)$. In Figs. 4(a) and 4(b), we show $F_{r}\left(r^{\prime}\right)$ and $F_{\phi}\left(r^{\prime}\right)$ for different values of $|\mathbf{E}|$ and $\alpha$ and $\Delta$. One can see that despite significant variations in $|\mathbf{E}|$ and $\alpha$ [Fig. 4(a)] or $\Delta$ [Fig. 4(b)], the points from different data sets cluster around well-defined functions.

The function $F_{r}$ has a pronounced biphasic shape. At distances larger than the effective core radius $\left(r^{\prime}>\lambda / 2 \pi\right)$, $F_{r}$ is very small. As $r^{\prime}$ decreases, $F_{r}$ grows (positive values correspond to repulsion). After reaching a maximum $\left(F_{r}^{\max }\right), F_{r}$ declines, and then it changes sign and becomes attractive. Around $r^{\prime}=0.1$, the attraction grows very rapidly (we only show an extrapolation of $F_{r}$ for these small values of $r^{\prime}$ because the attractive force grows rapidly as the core approaches the boundary of inclusion which makes its accurate assessment in our simulations difficult).

The function $F_{\phi}$ is biphasic as well; however, the positive values of $F_{\phi}$, indicating $\mathrm{CCW}$ orbital drift around the inclusion, are very small. The zero crossing (transition to CW motion) occurs around $r^{\prime}=0.15$. It is important to note that the dense clustering of the experimental points from different trajectories in Figs. 4(a) and 4(b) not only demonstrates the validity of Eqs. (3) and (4) but also has an important practical implication: for a given $\rho$, both $F_{r}$ and $F_{\phi}$ can be reconstructed from a single anchoring trajectory.

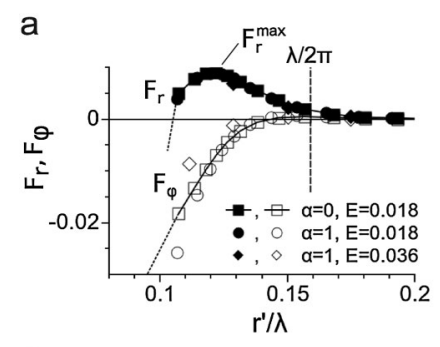

C
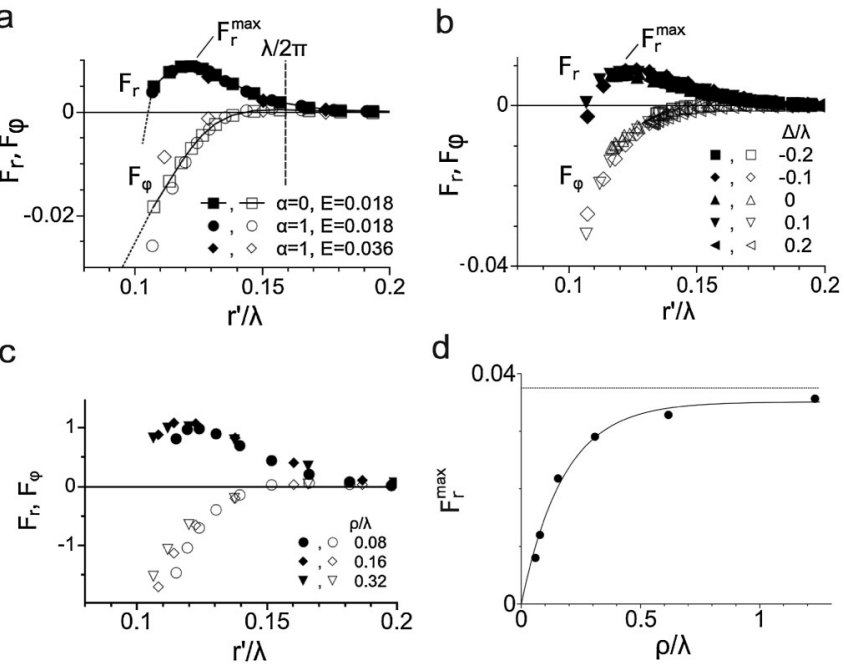

FIG. 4. Reconstructed radial and azimuthal forces. (a) $F_{r}$ (solid symbols) and $F_{\phi}$ (empty symbols) for drift generated for different values of $\alpha$ and $\mathbf{E}$. The dotted lines show extrapolations of $F_{r}$ and $F_{\phi}$ into the region close to the inclusion. The vertical dashed line indicates the effective core radius $\lambda / 2 \pi$. (b) $F_{r}$ and $F_{\phi}$ for different values of $\Delta$. (c) $F_{r}$ and $F_{\phi}$ for different $\rho$, normalized so that $F_{r}^{\max }$ is 1 . (d) Scaling function $S(\rho)$ [see Eq. (4)], which also gives the maximal repulsion force as a function of $\rho$. Dotted line shows $F_{r}^{\max }(\rho=\infty)$.

Our next step was to explore the dependence of $F_{r}$ and $F_{\phi}$ on the inclusion size $\rho$. We found that while $F_{r}$ and $F_{\phi}$ do depend on $\rho$, this dependence can be separated from that on $r^{\prime}$ by

$$
\begin{gathered}
F_{r}\left(r^{\prime}, \rho\right)=\tilde{F}_{r}\left(r^{\prime}\right) F_{r}^{\max }(\rho) \\
F_{\phi}\left(r^{\prime}, \rho\right)=\tilde{F}_{\phi}\left(r^{\prime}\right) F_{r}^{\max }(\rho) .
\end{gathered}
$$

The functions $\tilde{F}_{r}$ and $\tilde{F}_{\phi}$ are shown in Fig. 4(c) for three different values of $\rho$, ranging from $0.08 \lambda$ to $0.32 \lambda$. The fact that data points overlap for different values of $\rho$ shows that the variables $r^{\prime}$ and $\rho$ can indeed be separated as Eqs. (6) and (7) suggest.

We determined $F_{r}^{\max }$ for different values of $\rho$ [see Fig. 4(d)]. One can see that $F_{r}^{\max }(\rho)$ appears to be a simple exponential function

$$
F_{r}^{\max }(\rho) \approx F_{r}^{\max }(\infty)(1-\exp (-\beta \rho)),
$$

where $F_{r}^{\max }(\infty)$ is the maximum of $F_{r}$ at $\rho=\infty$. Note that to find $\beta$ and $F_{r}^{\max }\left({ }_{\infty}\right)$ and thus to fully define $F_{r}^{\max }(\rho)$, one needs to determine $F_{r}^{\max }$ for only two values of $\rho$. It is interesting that the empirically derived value of $\beta=0.177 \lambda$ is close to effective core radius $\lambda / 2 \pi=$ $0.159 \lambda$, which means that the effect of obstacles with the radius larger than the effective core size exert the force that is close to the one produced by an infinite wall.

The shape of $F_{r}$ and $F_{\phi}$ and their dependence on $\rho$ fully explains the complex dynamic behavior illustrated in 


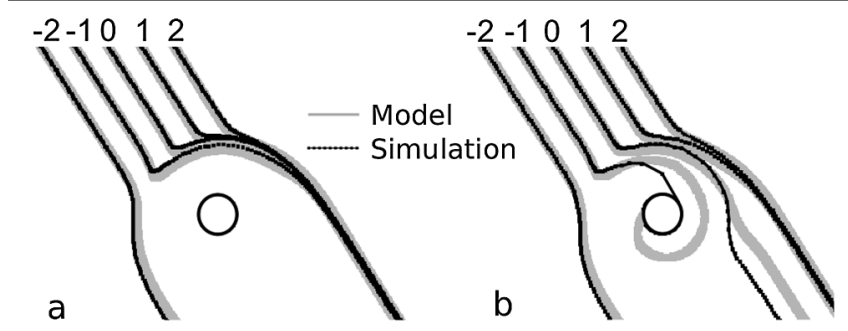

FIG. 5. Drift trajectories calculated using Eqs. (3) and (4) and reconstructed $F_{r}$ and $F_{\phi}$. Predicted trajectories (gray) versus true trajectories (black) for $\left|\mathbf{v}_{\mathbf{E}}\right|=6.8 \times 10^{-3} \lambda / T$ (a) and $\left|\mathbf{v}_{\mathbf{E}}\right|=$ $9.1 \times 10^{-3} \lambda / T$ (b). Trajectory labels $(-2, \ldots, 2)$ indicate the $\Delta$ of the trajectory in units of $0.12 \lambda$.

Figs. 1-3. One of the key factors is the existence of a repulsive barrier determined by $F_{r}^{\max }$. If the drift velocity of an approaching spiral is not large enough to overcome this barrier, the spiral gets deflected. For smaller obstacles the repulsive barrier is lower and the same drift velocity can be sufficient to overcome this barrier and anchor. The chirality-dependent bias in impact parameter illustrated in Fig. 3(b) is the result of the chiral azimuthal force $F_{\phi}$, which deflects the spiral from its central collision course and at short distances can be as strong if not stronger than $F_{r}$.

The drift trajectories predicted from the phenomenological Eqs. (3) and (4) are in good agreement with the experimental results obtained by directly solving the reaction-diffusion Eqs. (1) and (2) for different values of $|\mathbf{E}|$ and different initial conditions. The differences are noticeable only for trajectories near the separatrix between anchoring solutions and solutions that pass the inclusion on the right [see Fig. 5(b)], which is not surprising given the sensitivity of such trajectories to initial conditions.

It should be noted that the dynamic behavior described above is dependent on the excitability of the system, which in our particular case is determined by parameters $a$ and $b$ of Eq. (1). Specifically, the dynamic behaviors that we described here are observed for the "contracting scroll" region of parameter space [23]. We tested other points within this parameter range, and they showed very similar results.

We would like to emphasize that Eq. (3) is an approximation and has its limitations. The invariance of $F_{r}$ and $F_{\phi}$ is not perfect, which can be seen from careful examination of Fig. 4. This discrepancy may have the following physical explanation. A spiral wave senses the presence of an inclusion through the perturbation of the spiral's wave front that the inclusion creates. This perturbation propagates tangentially to the core thus creating a time lag $\tau$ between the moment the spiral reaches a certain location and the moment it receives the signal from the obstacle. We noticed that $F_{r}$ and $F_{\phi}$ vary much less if we use the location of the spiral during the previous rotation cycle to calculate the forces, i.e., $d \mathbf{x} / d t=\mathbf{v}_{\mathbf{E}}+\mathbf{F}\left(r^{\prime}(t-T), \rho\right)$; this time-lag correction was applied in Fig. 4. Another limitation of our method is the insufficient resolution at very small distances from the obstacles. In principle, a better resolution can be achieved by using much smaller inclusion sizes and respectively much smaller $E$ and drift speeds. Such simulations involve significant reduction of discretization steps and increase of computational costs which will require a dedicated effort.

In summary, our study has two main results. (i) We have introduced and tested a novel phenomenological approach for measuring forces exerted by an inclusion onto a spiral in an excitable medium. Our approach does not require the knowledge of dynamical equations of the system and can be applied to any experimental system with any type of inclusions or parametric heterogeneities. The independence of $F_{r}$ and $F_{\phi}$ of $\mathbf{E}$ as well as the simple scaling rule in Eqs. (6) and (7) suggest that the only experimental information required to fully define Eq. (3) are two anchoring trajectories for different inclusion sizes. (ii) We have advanced the understand of anchoring to impermeable inclusions, a special class of heterogeneities that are important for cardiological applications. We discovered that spiral and scroll waves can be repelled by such inclusions and that repulsion increases with the size of the inclusion. Another unexpected result was significant involvement of chiral forces that favor anchoring during off-center collisions. These findings improve the understanding of the interaction of drifting scroll waves with impermeable inclusions and represent an important step toward understanding scroll wave dynamics in the heart.

[1] M. C. Cross and P. C. Hohenberg, Rev. Mod. Phys. 65, 851 (1993).

[2] J. M. Davidenko, A. V. Pertsov, R. Salomonsz, W. Baxter, and J. Jalife, Nature (London) 355, 349 (1992).

[3] A. N. Zaikin and A.M. Zhabotinsky, Nature (London) 225, 535 (1970).

[4] A. T. Winfree, Science 175, 634 (1972).

[5] A. T. Winfree, Science 181, 937 (1973).

[6] J. P. Keener, Physica (Amsterdam) 31D, 269 (1988).

[7] V. N. Biktashev, A. V. Holden, and H. Zhang, Phil. Trans. R. Soc. A 347, 611 (1994).

[8] A. Rudenko and A. Panfilov, Studia Biophysica 98, 183 (1983).

[9] M. Wellner, A. M. Pertsov, and J. Jalife, Phys. Rev. E 59, 5192 (1999).

[10] E. A. Ermakova, A. M. Pertsov, and E. E. Shnol, Physica (Amsterdam) 40D, 185 (1989).

[11] R. M. Zaritski and A. M. Pertsov, Phys. Rev. E 66, 066120 (2002).

[12] C. Zemlin, K. Mukund, M. Wellner, R. Zaritsky, and A. Pertsov, Phys. Rev. Lett. 95, 098302 (2005).

[13] A. Pertsov, E. Ermakova, and A. Panfilov, Physica (Amsterdam) 14D, 117 (1984).

[14] X. Zou, H. Levine, and D. A. Kessler, Phys. Rev. E 47, R800 (1993).

[15] M. Vinson, A. Pertsov, and J. Jalife, Physica (Amsterdam) 72D, 119 (1994). 
[16] V. N. Biktashev, D. Barkley, and I. V. Biktasheva, Phys. Rev. Lett. 104, 058302 (2010).

[17] D. Pazó, L. Kramer, A. Pumir, S. Kanani, I. Efimov, and V. Krinsky, Phys. Rev. Lett. 93, 168303 (2004).

[18] A. Pumir, S. Sinha, S. Sridhar, M. Argentina, M. Hörning, S. Filippi, C. Cherubini, S. Luther, and V. Krinsky, Phys. Rev. E 81, 010901 (2010).

[19] S. Takagi, A. Pumir, D. Pazó, I. Efimov, V. Nikolski, and V. Krinsky, Phys. Rev. Lett. 93, 058101 (2004).
[20] I. V. Biktasheva, D. Barkley, V.N. Biktashev, and A.J. Foulkes, Phys. Rev. E 81, 066202 (2010).

[21] V. N. Biktashev, I. V. Biktasheva, and N. A. Sarvazyan, PLoS ONE 6, e24388 (2011).

[22] D. Barkley, M. Kness, and L. S. Tuckerman, Phys. Rev. A 42, 2489 (1990).

[23] S. Alonso, F. Sagués, and A. S. Mikhailov, Science 299, 1722 (2003). 